\title{
Contrôle Physicochimique des Feuilles de Lippia chevalieri Moldenke Cultivé
}

\author{
Mamadou Lamine Diarra, \\ Mahamane Haïdara, \\ Adama Dénou, Sékou Doumbia, \\ Daouda Dembélé, \\ Drissa Diallo,
}

Faculté de Pharmacie, Université des Sciences, des Techniques et des

Technologies de Bamako, Bamako, Mali

Seydou Mamadou Dembélé, N'golo Ballo, Rokia Sanogo,

Département de Médecine Traditionnelle (DMT), Institut National de

Recherche en Santé Publique (INRSP), Bamako, Mali

Kandioura Noba,

Laboratoire de Botanique et Biodiversité,

Université Cheikh Anta Diop de Dakar, Fann, Sénégal

Doi:10.19044/esj.2019.v15n33p274 URL:http://dx.doi.org/10.19044/esj.2019.v15n33p274

\section{Résumé}

Au Mali, le Malarial 5 qui est un Médicament Traditionnel Amélioré (MTA) produit en partie à base de feuilles de Lippia chevalieri est utilisé pour traiter les symptômes du paludisme simple. Les feuilles de Lippia chevalieri cultivé peuvent être utilisées pour la préparation des MTA lorsqu'elles sont de qualité. La présente étude portée sur le contrôle physicochimique a montré que les feuilles de Lippia chevalieri cultivé ont des teneurs en eau inférieures à $10 \%$, celles des cendres totales un peu élevées et celles des cendres insolubles dans l'acide chlorhydriques à 10\% faibles. Les meilleurs rendements des extractions sont obtenus par l'eau par infusion et par décoction. Plusieurs groupes chimiques comprenant entre autres des caroténoïdes, des coumarines, des mucilages, des flavonoïdes ont été révélés.

Mots-clés: Lippia chevalieri, Teneur en eau, Teneur en cendres, Substances extractibles, Composition chimique 


\title{
Physicochemical Control of Leaves of Cultivated Lippia chevalieri Moldenke
}

\author{
Mamadou Lamine Diarra, \\ Mahamane Haïdara, \\ Adama Dénou, Sékou Doumbia, \\ Daouda Dembélé, \\ Drissa Diallo,
}

Faculté de Pharmacie, Université des Sciences, des Techniques et des

Technologies de Bamako, Bamako, Mali

Seydou Mamadou Dembélé, N'golo Ballo, Rokia Sanogo,

Département de Médecine Traditionnelle (DMT), Institut National de

Recherche en Santé Publique (INRSP), Bamako, Mali

Kandioura Noba,

Laboratoire de Botanique et Biodiversité,

Université Cheikh Anta Diop de Dakar, Fann, Sénégal

\begin{abstract}
In Mali, Malarial 5, which is an Improved Traditional Medicine (ITM) produced partly from the leaves of Lippia chevalieri, is used to treat the symptoms of uncomplicated malaria. The leaves of cultivated Lippia chevalieri can be used for the preparation of improved traditional medicines because they are of good quality. This paper focuses on the physicochemical control which shows that the leaves of cultivated Lippia chevalieri contain less than $10 \%$ of moisture content. The total ash content was a little higher, and the acid insoluble ash was low. The best yields of extractions are obtained with water through infusion and decoction. Several chemical compounds including carotenoids, coumarins, mucilage, and flavonoids were revealed.
\end{abstract}

Keywords: Lippia chevalieri, Moisture content, Ash content, Extractive substances, Chemical composition

\section{Introduction}

Lippia chevalieri est une Verbenaceae utilisée comme supplément alimentaire pendant la grossesse (Nergard et al., 2015). Au Mali, il entre dans 
la préparation d'un Médicament Traditionnel Amélioré (MTA) appelé Malarial 5 (Willcox et al., 2012). Utilisé pour le traitement du paludisme simple, le Malarial 5 est sur la liste nationale des médicaments essentiels du Mali (Ministère de la Santé, 2005).

La matière première utilisée pour la préparation de ce MTA est constituée par les feuilles de Lippia chievalieri et de Senna occidentalis et les capitules de Acmella oleracea. Cette matière première est cueillie dans des peuplements naturels. La disponibilité de matière première en raison des contraintes climatiques et anthropiques, ne permet plus d'assurer un approvisionnement régulier pour la production de ce MTA (MEATEU, 2000). La culture des plantes médicinales permet de satisfaire les besoins des pays en matières premières. Ces matières premières ne peuvent être utilisées pour la préparation des médicaments traditionnels améliorés que quand elles sont de qualité (Guo et al., 2009). La présente étude a été entreprise pour contrôler la qualité physicochimique des feuilles des plants cultivés de Lippia chevalieri.

\section{Matériel et méthodes}

\subsection{Matériel végétal}

Le matériel végétal a été constitué par des poudres des feuilles des plants sauvages et cultivés de Lippia chevalieri. Les plants cultivés ont reçu trois traitements de fertilisation. Le premier traitement était le non apport de nutriment végétal $\left(\mathrm{T}_{0}\right)$, le deuxième l'apport de nutriment à $5 \mathrm{t} / \mathrm{ha}\left(\mathrm{T}_{1}\right)$ et le troisième l'apport de nutriment à $10 \mathrm{t} / \mathrm{ha}\left(\mathrm{T}_{2}\right)$. Les feuilles étaient issues des plants cultivés récoltés 55 jours après repiquage (JAR), 73 JAR, 95 JAR, 116 JAR 161 JAR et de 224 JAR. Une seule récolte a été réalisée avec les plants sauvages.

\subsection{Méthodes utilisées}

La méthode consistait à la détermination de la teneur en eau, de la teneur en cendres totales et insolubles dans l'acide chlorhydrique à 10\%, des substances extractibles par les solvants, l'indice de mousse et les groupes chimiques présents dans des échantillons de poudre de feuilles de Lippia chevalieri sauvages et cultivés. Les solvants utilisés étaient l'eau, l'éthanol 30 et $70 \%$ et l'éther de pétrole.

\section{Pulvérisation}

Les feuilles ont été pulvérisées en poudre fine au mortier traditionnel.

\section{Teneur en eau}

Une quantité de 2 à $3 g$ de poudre (masse prise d'essai) dans un verre de montre préalablement taré, a été placée dans l'étuve à $105^{\circ} \mathrm{C}$ pendant 24 heures. La perte en poids a été déterminée après 24 heures après dessiccation. 
Le test a été répété quatre à cinq fois et la moyenne a été déterminée. Le pourcentage en eau a été calculé par les formules suivantes:

Masse prise d'essai = masse avant étuve - tare

Masse eau = masse avant étuve - masse après étuve

$$
\% \text { en eau }=\frac{\text { Masse eau }}{\text { Masse prise d'essai }} \times 100
$$

La tare est la masse du verre de montre. La masse avant étuve est celle de la prise d'essai avant dessiccation et celle du verre de montre. La masse après étuve est celle de la prise d'essai après dessiccation et celle du verre de montre.

\section{Teneur en cendres totales}

Trois prises d'essai de la poudre utilisée pour la teneur en eau ont été pesées dans 3 creusets en porcelaine préalablement taré $(\mathrm{M})$. Après incinération au four à une température d'environ $600{ }^{\circ} \mathrm{C}$ pendant 6 heures, et refroidissement dans un dessiccateur, les masses ( $\left.M^{\prime}\right)$ des creusets contenant les cendres ont été déterminées.

La masse des cendres totales (Mct) contenue dans le creuset et la masse de la prise d'essai (Mpe) sont déterminées par les formules suivantes :

$$
\text { Mct }=\mathrm{M}^{\prime}-\mathrm{T} \quad \text { Mpe }=\mathrm{M}-\mathrm{T}
$$

La tare (T) est la masse du creuset en porcelaine. La masse de la prise d'essai (Mpe) est celle des prises d'essai utilisée pour la teneur en eau avant incinération moins celle des creusets. La masse des cendres totales (Mct) est celle des prises d'essai utilisée pour la teneur en eau après incinération moins celle des creusets.

$$
\% \mathrm{Ct}=\frac{\mathrm{Mct}}{\mathrm{Mpe}} \quad \mathrm{x} \quad 100
$$

Calculer le pourcentage des cendres totales $(\% \mathrm{Ct})$ par la formule suivante:

\section{Teneur en cendres insolubles dans l'acide chlorhydrique à $10 \%$}

Les cendres totales préalablement obtenues ont été bouillies dans 20 $\mathrm{ml}$ d'acide chlorhydrique et filtrées sur du papier sans cendre. Ce papier a été transféré dans un creuset sec déjà taré de masse M. L'ensemble est pesé ( $\left.M^{\prime}\right)$, incinéré et repesé ( $\mathrm{M}$ ')

La masse des cendres chlorhydriques (MCc) est donnée par la formule suivante :

$$
\mathrm{MCc}=\mathrm{M}^{\prime}-\mathrm{M}^{\prime},
$$

Calculer le pourcentage des cendres chlorhydriques $(\% \mathrm{Cc})$ par la formule suivante : 
MCc

$\% \mathrm{Cc} \quad \mathrm{x} \quad 100$

$\sum$ Mpe est la somme des masses de prise d'essai pour la détermination de la teneur en eau.

\section{Substances extractibles}

Le Dosage des substances extractibles par l'eau a été effectué par infusion et décoction et celui des substances extractibles par l'éther de pétrole, l'éthanol à $30 \%$ et $70 \%$ par macération de 24 heures.

\section{Eau}

\section{Infusé}

$1 \mathrm{~g}$ de poudre a été extrait avec $20 \mathrm{ml}$ d'eau par infusion. Après filtration, l'infusé a été évaporé à sec à $105^{\circ} \mathrm{C}$ dans un creuset préalablement taré. Soit $\left(\mathrm{M}_{1}\right)$ la masse du creuset vide et $\left(\mathrm{M}_{2}\right)$ la masse du creuset avec l'extrait sec, le pourcentage de substances extractibles est calculé comme suit :

$$
\% \mathrm{Ct}=\frac{\text { Mct }}{\text { Mpe }} \times 100
$$

\section{Décocté}

$1 \mathrm{~g}$ de poudre a été extrait avec $20 \mathrm{ml}$ d'eau par décoction. Après filtration, le décocté a été évaporé à sec dans un creuset préalablement taré. Soit $\left(\mathrm{M}_{1}\right)$ la masse du creuset vide et $\left(\mathrm{M}_{2}\right)$ la masse du creuset avec l'extrait sec, le pourcentage de substances extractibles est calculé comme suit :

$$
\% \text { substances extractibles }=\frac{\mathrm{M}_{2}-\mathrm{M}_{1}}{1} \times 100
$$

\section{Ethanol à $30 \%$ et $70 \%$}

$5 \mathrm{~g}$ de poudre a été extrait avec $100 \mathrm{ml}$ d'éthanol à $30 \%$ et $70 \%$ par macération de 24 heures. Après filtration, le filtrat a été évaporé à sec dans un creuset préalablement taré. Soit $\left(\mathrm{P}_{1}\right)$ la masse du creuset vide et $\left(\mathrm{P}_{2}\right)$ la masse du creuset avec l'extrait sec, le pourcentage de substances extractibles est calculé comme suit :

$$
\% \text { substances extractibles }=\frac{\mathrm{M}_{2}-\mathrm{M}_{1}}{1} \times 100
$$

\section{Ether de pétrole}

$5 \mathrm{~g}$ de poudre a été extrait avec $100 \mathrm{ml}$ d'éther de pétrole par macération de 24 heures. Après filtration, le filtrat a été évaporé à sec dans un creuset préalablement taré. Soit $\left(\mathrm{N}_{1}\right)$ la masse du creuset vide et $\left(\mathrm{N}_{2}\right)$ la masse 
du creuset avec l'extrait sec, le pourcentage de substances extractibles par l'éther est calculé comme suit :

$$
\% \text { substances extractibles par l'éther }=\frac{\mathrm{N}_{2}-\mathrm{N}_{1}}{5} \times 100
$$

\section{Détermination des groupes chimiques}

Les alcaloïdes, les anthocyanes, les anthracénosides, les caroténoïdes, les composés réducteurs, les coumarines, les flavonoïdes, les hétérosides cardiotoniques, les hétérosides cyanogénétiques, les leuco-anthocyanes, les mucilages, les oses et les holosides, les tanins, les stérols, les triterpènes et les saponosides avec leur indice de mousse ont été recherchés dans des feuilles sauvages et cultivés de Lippia chevalieri par les réactions colorées et de précipitation en tube et par chromatographie sur couche mince (CCM).

\section{Résultats}

Les teneurs en eau des poudres de feuilles ont été inférieures à $10 \%$ aussi bien pour les plants sauvages que cultivés (Tableau 1). Les teneurs en cendres totales étaient de $21,11 \%$ pour les plants sauvages et $19,97 \%$ en moyenne pour les plants cultivés (Tableau 1).

Les cendres insolubles dans l'acide chlorhydrique à $10 \%$ avec 5,33\% pour les plants sauvages et $5,36 \%$ de moyenne pour les plants cultivés, étaient plus faibles par rapport aux cendres totales (Tableau 1).

Tableau 1. Teneur en eau, en cendres totales (CT) et en cendres insolubles dans l'acide chlorhydrique à $10 \%(\mathrm{CHCl})$ des feuilles séchées de plants sauvages et cultivés de Lippia chevalieri.

\begin{tabular}{lllll}
\hline Récoltes & Traitéments & $\begin{array}{l}\text { Teneur en eau } \\
(\boldsymbol{\%})\end{array}$ & Teneur en $\mathbf{C}_{\mathbf{T}} \mathbf{( \% )}$ & $\begin{array}{l}\text { Teneur en } \mathbf{C}_{\mathbf{H C l}} \\
(\boldsymbol{\%})\end{array}$ \\
\hline \multirow{3}{*}{ 55 JAR } & $\mathrm{T}_{0}$ & 7,84 & 18,33 & 4,35 \\
& $\mathrm{~T}_{1}$ & 8,53 & 17,76 & 4,70 \\
& $\mathrm{~T}_{2}$ & 8,15 & 18,46 & 4,94 \\
& $\mathrm{~T}_{0}$ & 8,75 & 20,33 & 6,38 \\
$\mathbf{7 3}$ JAR & $\mathrm{T}_{1}$ & 4,12 & 17,70 & 3,25 \\
& $\mathrm{~T}_{2}$ & 5,86 & 21,86 & $\mathbf{7 , 2 3}$ \\
& $\mathrm{T}_{0}$ & 5,49 & 20,06 & 4,24 \\
95 JAR & $\mathrm{T}_{1}$ & 5,36 & 17,80 & 3,87 \\
& $\mathrm{~T}_{2}$ & 7,78 & 18,74 & 5,55 \\
\multirow{3}{*}{ 116 JAR } & $\mathrm{T}_{0}$ & 7,70 & 18,53 & 5,34 \\
& $\mathrm{~T}_{1}$ & 7,46 & 18,57 & 5,22 \\
& $\mathrm{~T}_{2}$ & 8,22 & 19,81 & 5,48 \\
\multirow{3}{*}{ 161 JAR } & $\mathrm{T}_{0}$ & 6,99 & 21,91 & 6,12 \\
& $\mathrm{~T}_{1}$ & 6,85 & 21,22 & 5,85 \\
\multirow{2}{*}{ 224 JAR } & $\mathrm{T}_{2}$ & 6,48 & 23,25 & 6,85 \\
& $\mathrm{~T}_{0}$ & 6,62 & $\mathbf{2 3 , 3 7}$ & 6,12 \\
\hline & $\mathrm{T}_{1}$ & 9,63 & 21,28 & 5,38 \\
\hline
\end{tabular}




\begin{tabular}{lllll}
\hline \multirow{2}{*}{ Ps } & $\mathrm{T}_{2}$ & $\mathbf{9 , 8 6}$ & 20,52 & 5,62 \\
& $\mathrm{St}$ & 8,63 & 21,11 & 5,33 \\
\hline
\end{tabular}

JAR : Jour après repiquage ; NS : Non significatif ; Ps : Plants sauvages, St : Sans traitement.

Le rendement le plus élevé par infusion a été obtenu avec les plants cultivés à la récolte de $161 \mathrm{JAR}\left(\mathrm{T}_{2}\right)$ avec $37 \%$. Il était de $24 \%$ avec les plants sauvages (Tableau 2). Par décoction, le meilleur rendement a été obtenu avec les plants cultivés à la récolte de $224 \mathrm{JAR}\left(\mathrm{T}_{0}\right)$ avec $48 \%$. Il était de $24 \%$ avec les plants sauvages (Tableau 2).

Tableau 2. Teneur en substances extraites des feuilles séchées de plants sauvages et cultivés de Lippia chevalieri par l'eau par infusion et par décoction.

\begin{tabular}{|c|c|c|c|}
\hline Récoltes & Traitéments & $\begin{array}{l}\text { Teneur (\%) de } \\
\text { substances } \\
\text { extraites par } \\
\text { infusion } \\
\end{array}$ & $\begin{array}{l}\begin{array}{l}\text { Teneur (\%) de } \\
\text { substances } \\
\text { extraites par } \\
\text { décoction }\end{array} \\
\end{array}$ \\
\hline \multirow{3}{*}{55 JAR } & $\mathrm{T}_{0}$ & 17 & 26 \\
\hline & $\mathrm{T}_{1}$ & 14 & 22 \\
\hline & $\mathrm{T}_{2}$ & 13 & 26 \\
\hline \multirow{3}{*}{73 JAR } & $\mathrm{T}_{0}$ & 18 & 24 \\
\hline & $\mathrm{T}_{1}$ & 26 & 34 \\
\hline & $\mathrm{T}_{2}$ & 21 & 28 \\
\hline \multirow{3}{*}{95 JAR } & $\mathrm{T}_{0}$ & 16 & 26 \\
\hline & $\mathrm{T}_{1}$ & 16 & 26 \\
\hline & $\mathrm{T}_{2}$ & 14 & 24 \\
\hline \multirow{3}{*}{116 JAR } & $\mathrm{T}_{0}$ & 17 & 22 \\
\hline & $\mathrm{T}_{1}$ & 24 & 30 \\
\hline & $\mathrm{T}_{2}$ & 27 & 34 \\
\hline \multirow{3}{*}{161 JAR } & $\mathrm{T}_{0}$ & 28 & 36 \\
\hline & $\mathrm{T}_{1}$ & 35 & 44 \\
\hline & $\mathrm{T}_{2}$ & 37 & 38 \\
\hline \multirow{3}{*}{224 JAR } & $\mathrm{T}_{0}$ & 33 & 48 \\
\hline & $\mathrm{T}_{1}$ & 24 & 36 \\
\hline & $\mathrm{T}_{2}$ & 25 & 36 \\
\hline Ps & $\mathrm{St}$ & 24 & 24 \\
\hline
\end{tabular}

Les meilleurs rendements avec l'éthanol à $30 \%$ et $70 \%$ ont été obtenus avec les plants sauvages avec respectivement $11 \%$ et $13 \%$. Avec l'éther de pétrole le meilleur rendement a été obtenu avec les plants cultivés à la récolte de 73 JAR $\left(\mathrm{T}_{2}\right)$ avec 12\% (Tableau 3 ). 
Tableau 3. Teneur en substances extraites (\%) des feuilles séchées de plants sauvages et cultivés de Lippia chevalieri par l'éthanol à 30\%,70\% et l'éther de pétrole par macération.

\begin{tabular}{|c|c|c|c|c|}
\hline Récoltes & Traitéments & $\begin{array}{lr}\text { Teneur }(\%) \\
\text { de substances } \\
\text { extraites } \\
\text { Ethanol } \\
30 \%\end{array}$ & $\begin{array}{l}\text { Teneur }(\%) \\
\text { de substances } \\
\text { extraites par } \\
\text { Ethanol à } \\
70 \%\end{array}$ & $\begin{array}{lr}\text { Teneur } & (\%) \\
\text { de substances } \\
\text { extraites par } \\
\text { Ether } \\
\text { pétrole }\end{array}$ \\
\hline \multirow{3}{*}{55 JAR } & $\mathrm{T}_{0}$ & 2 & 4 & 10 \\
\hline & $\mathrm{T}_{1}$ & 3 & 4 & 10 \\
\hline & $\mathrm{T}_{2}$ & 6 & 8 & 8 \\
\hline \multirow{3}{*}{73 JAR } & $\mathrm{T}_{0}$ & 4 & 6 & 6 \\
\hline & $\mathrm{T}_{1}$ & 3 & 4 & 4 \\
\hline & $\mathrm{T}_{2}$ & 2 & 4 & 12 \\
\hline \multirow{3}{*}{95 JAR } & $\mathrm{T}_{0}$ & 2 & 4 & 4 \\
\hline & $\mathrm{T}_{1}$ & 5 & 6 & 4 \\
\hline & $\mathrm{T}_{2}$ & 2 & 4 & 2 \\
\hline \multirow{3}{*}{116 JAR } & $\mathrm{T}_{0}$ & 6 & 8 & 6 \\
\hline & $\mathrm{T}_{1}$ & 3 & 4 & 4 \\
\hline & $\mathrm{T}_{2}$ & 8 & 10 & 4 \\
\hline \multirow{3}{*}{161 JAR } & $\mathrm{T}_{0}$ & 1 & 2 & 2 \\
\hline & $\mathrm{T}_{1}$ & 2 & 2 & 2 \\
\hline & $\mathrm{T}_{2}$ & 3 & 4 & 2 \\
\hline \multirow[t]{3}{*}{224 JAR } & $\mathrm{T}_{0}$ & 1 & 2 & 2 \\
\hline & $\mathrm{T}_{1}$ & 2 & 4 & 4 \\
\hline & $\mathrm{T}_{2}$ & 2 & 2 & 2 \\
\hline Ps & & 11 & 13 & 2 \\
\hline
\end{tabular}

JAR : Jour après repiquage ; NS : Non significatif ; Ps : Plants sauvages, St : Sans traitement.

Les réactions de caractérisation en tube et par CCM des plants sauvages et cultivés ont montré la présence de caroténoïdes, de coumarines, de mucilages, de saponosides, de stérols, de triterpènes, de tanins et de flavonoides (Tableau 4). Les indices de mousse étaient inférieurs à 100.

Tableau 4. Résultat des réactions de caractérisation des feuilles séchées de plants sauvages et cultivés de Lippia chevalieri.

\begin{tabular}{lll}
\hline $\begin{array}{l}\text { Groupes } \\
\text { chimiques }\end{array}$ & Traitéments & Résultats \\
\hline \multirow{4}{*}{ Alcalö̈des } & $\mathrm{T}_{0}$ & - \\
& $\mathrm{T}_{1}$ & - \\
& $\mathrm{T}_{2}$ & - \\
& $\mathrm{Pt}$ & - \\
\multirow{4}{*}{ Anthocyanes } & $\mathrm{T}_{0}$ & - \\
& $\mathrm{T}_{1}$ & - \\
& $\mathrm{T}_{2}$ & - \\
& $\mathrm{Pt}$ & - \\
\multirow{4}{*}{ Antracénosides } & $\mathrm{T}_{0}$ & - \\
& $\mathrm{T}_{1}$ & - \\
& $\mathrm{T}_{2}$ & - \\
& $\mathrm{Pt}$ & - \\
\hline
\end{tabular}




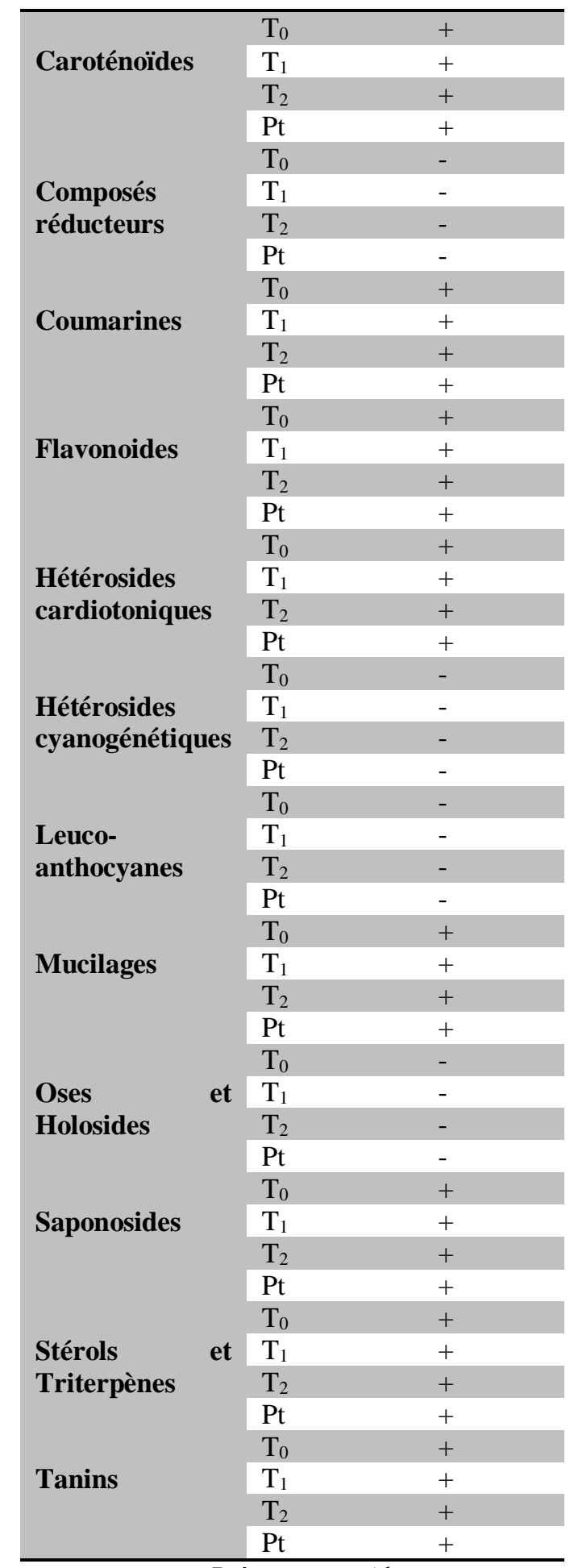

$+:$ Présence ; - : Absence 


\section{Discussion}

Les teneurs en eau inférieures à $10 \%$ traduisent le bon séchage de la matière première. Une teneur supérieure à $10 \%$ a été obtenue avec Lippia multiflora après séchage (Konan et al., 2010). Les teneurs en cendres totales étaient $21,11 \%$ pour les plants sauvages et $19,97 \%$ en moyenne pour les plants cultivés, Konan a obtenu une teneur plus faible avec les feuilles séchées de Lippia multiflora (Konan et al., 2010). Donc les feuilles de Lippia chevalieri sont plus riches en substances minérales que celles de Lippia multiflora.

Les teneurs faibles en cendres insolubles dans l'acide chlorhydrique à $10 \%$ indiquent l'absence d'impuretés dans la matière première au cours des récoltes et du séchage aussi bien pour les plants sauvages que cultivés.

Les meilleurs rendements des extractions ont été obtenus avec l'eau par infusion et par décoction avec les plants cultivés. Ce résultat justifie aussi la forme d'utilisation du Malarial 5 qui est la décoction (Ministère de la Santé, 2005).

Parmi les groupes chimiques révélés, les tanins, les saponosides et les stérols avaient été signalés par Konaté (Konaté, 2005), les flavonoïdes avait été signalés par Bangou (Bangou et al., 2012).

\section{Conclusion}

Les poudres de feuilles de plants cultivés de Lippia chevalieri ont des teneurs en eau inférieures à $10 \%$, celles des cendres totales sont un peu élevées et celles des cendres insolubles dans l'acide chlorhydriques à 10\% sont faibles. Les meilleurs rendements des extractions des plants cultivés sont obtenus avec eau par infusion et par décoction.

Les groupes chimiques révélés dans les plants cultivés comprennent des caroténoïdes, des coumarines, des mucilages, des saponosides, des stérols, des triterpènes, des tanins et des flavonoïdes. Les indices de mousses sont inférieurs à 100.

En conclusion, ces résultats constituent les éléments de contrôle physicochimique des feuilles de Lippia chevalieri.

\section{Conflit d'intérêts}

Il n'y a pas eu de conflit d'intérêts.

\section{Contributions des Auteurs}

MLD a conçu, conduit l'étude et participé à la rédaction de l'article. AD, $\mathrm{MH}, \mathrm{SD}, \mathrm{DD}, \mathrm{SMD}$, et NB ont participé à la rédaction de l'article. RS, DD et $\mathrm{KN}$ ont relu et corrigé l'article. 


\section{Remerciements}

Les auteurs de ce travail adressent leur profonde gratitude au personnel du Département de Médecine Traditionnelle de l'INRSP.

\section{References:}

1. Bangou, MJ., Norma, AA., Meda, RNT., Yougbaré, ZM., MillogoRasolodimby, J. \& Nacoulma, OG. (2012). Lippia chevalieri Moldenke: A brief review of traditional uses, phytochemistry and pharmacology. International Journal of Drug Delivery, 4, pp. 289296.

2. Guo, HB., Song, ZP., Liang, ZS. \& Zhang, YJ. (2009). Domestic cultivation may abate the contradiction between sustainable utilization and genetic diversity conservation of medicinal plants. Journal of Medicinal Plants Research, 3(13), pp. 1184-1188.

3. Konan, JLK., Turquin, L., Attah, H., Yao-Kouamé, A., Allou, K. \& Ake, S. (2010). Lippia multiflora (Verbenaceae) en Côte D'Ivoire : point des premiers résultats de recherche et enjeu cultural. Agronomie Africaine, 22(2), pp. 121-130.

4. Konaté, N. (2005). Etude de la consommation des médicaments traditionnels améliorés dans le cercle de Kadiolo. Thèse Pharmacie. FMPOS, Université de Bamako, Bamako, 178 p.

5. Ministère de l'Equipement de l'Aménagement du Territoire de l'Environnement et de l'Urbanisme (MEATEU) (2000). Situation générale de la diversité biologique au mali. Stratégie nationale en matière de diversité biologique, Tome1. Rapport, 103 p.

6. Ministère de la Santé (2005). Formulaire thérapeutique national, Mali, $477 \mathrm{p}$.

7. Nergard, CS., Ho, TPT., Diallo, D., Ballo, N., Paulsen, BS. \& Nordeng, H. (2015). Attitudes and use of medicinal plants during pregnancy among women at health care centers in three regions of Mali, West-Africa. Journal of Ethnobiology and Ethnomedicine, 11(73), pp. 1-11.

8. Willcox, M., Sanogo, R., Diakite, C., Giani, S., Paulsen, BS. \& Diallo, D. (2012). Improved traditional medicines in Mali. The Journal of Alternative and Complementary Medicine, 18, pp. 212-220. 